\title{
Familial anaplastic ependymoma: evidence of loss of chromosome 22 in tumour cells
}

\author{
P C G Nijssen, R H Lekanne Deprez, C C Tijssen, A Hagemeijer, E P J Arnoldus, \\ J L J M Teepen, R Holl, M F Niermeyer
}

\begin{abstract}
A family with anaplastic ependymomas, histologically verified in three cases and neuroradiologically suggested in a fourth, is presented. Two healthy brothers both had two affected sons. All four male patients were younger than 5 years at the time of diagnosis. Two boys died before the age of 3 years. Genotype analysis (with polymorphic DNA markers for chromosome 22 and interphase cytogenetic analysis) of one of the tumours showed a subpopulation of tumour cells with monosomy of (part of) chromosome 22. Non-neoplastic cells of this patient showed a normal karyotype. These findings give further evidence for the role of a tumour suppressor gene on chromosome 22 in the pathogenesis of familial ependymal tumours.
\end{abstract}

(F Neurol Neurosurg Psychiatry 1994;57:1245-1248)

Department of

Neurology

P C G Nijssen

C C Tijssen

Department of Neuropathology

J L J M Teepen

Department of

Pediatrics, St

Elisabeth Hospital,

Hilvarenbeekseweg 60,

Tilburg, The

Netherlands

R Holl

Department of

Pathology

R H Lekanne Deprez

Departments of Cell

Biology and Genetics

A Hagemeijer

Department of Clinical

Genetics, Erasmus

University,

Rotterdam,

The Netherlands

M F Niermeyer

University Hospital

of Leiden,

The Netherlands

E P J Arnoldus

Correspondence to:

Dr C C Tijssen, Department

of Neurology, St Elisabeth

Hospital, P O Box 90151,

5000 LC Tilburg, The

Netherlands.

Received 15 November 1993

and in revised form

10 March 1994.

Accepted 3 May 1994

Familial occurrence of brain tumours is infrequent, but reports exist, especially for medulloblastomas, glioblastomas, and astrocytomas. Concurrence of ependymal tumours in two sisters made up the first report on familial brain tumours, and subsequent familial ependymal tumours have been reported, although rarely. ${ }^{1}$

Cytogenetic and DNA studies on ependymal tumours are scarce. ${ }^{2}$ From the medical literature, deletions or translocations of chromosome 22 were found in 12 out of 30 analysed ependymomas. Monosomy 22 was found in one tumour of a family with two sisters and a maternal male cousin with ependymomas. ${ }^{3}$ We present a family with four male cousins whose fathers are brothers, with verified ependymal brain tumours in three cousins and neuroradiological evidence in the fourth.

\section{Family study}

Figure 1 shows the pedigree of the family. No signs of neurofibromatosis were present in patients $A$ to $D$, or in their relatives. The mothers of the patients were not related or consanguinous to their partners, and did not use medication during or around pregnancy. Spontaneous abortion occurred once in the mother of patients A and B, and four times in the mother of patients $C$ and D. A sister of the paternal grandfather of the affected children died at two years of age, after an operation for a brain tumour, but no medical or pathology records were available. Tracing of the family pedigree according to paternal links was possible back to 1689 . This showed several childhood deaths in each generation, possibly because of higher childhood mortality at that time. Cerebral MRI of the father of patients $C$ and $D$ was normal.

Patient A, born 1977, presented at the age of 5 years with headache, vomiting, somnolence, and right hemiataxia. Computed tomography showed a tumour in the fourth ventricle and right cerebellar hemisphere (fig 2A). Treatment consisted of tumour extirpation, and postoperative radiotherapy and chemotherapy (methotrexate, vincristine, and prednisolone). Hydrocephalus developed but the patient improved after drainage. $\mathrm{He}$ is now 15 years old and is well, except for moderate cognitive disturbances and slight ataxia.

Patient B, born 1981, a brother of patient

A the age of 3 days with irregular respiration, and slight icterus. Computed tomography did not allow a specific diagnosis because of low quality. Six months later, he was readmitted for drowsiness, vomiting, and weight loss. Computed tomography showed a large tumour in the fourth ventricle, with contrast enhancement and hydrocephalus

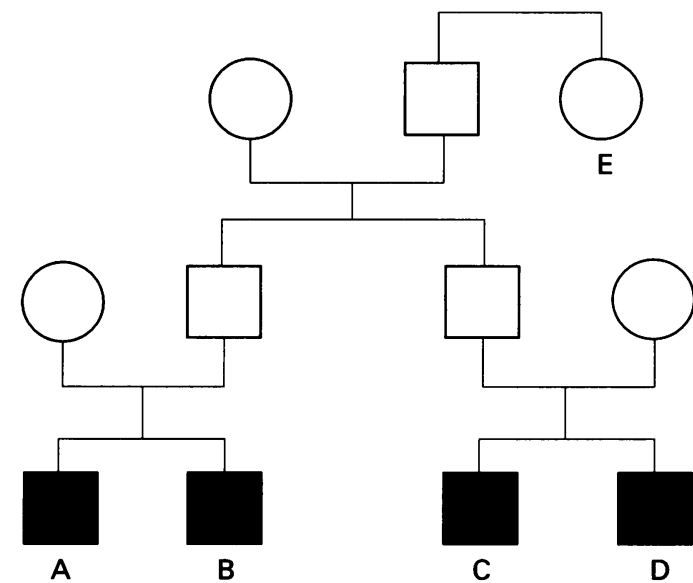

Figure 1 Family pedigree. Patients $A$ to $D$ had proved ependymal brain tumours; patient $E$ died two years after operation for a brain tumour (anecdotal). 
Figure 2 Cerebral scans. (A) CT of patient $A$, showing an infratentorial tumour with contrast enhancement. (B) CT of patient $B$. (C) MRI of patient $C$, showing a large supratentorial

intraventricular tumour.

(D) $C T$ of patient $D$.
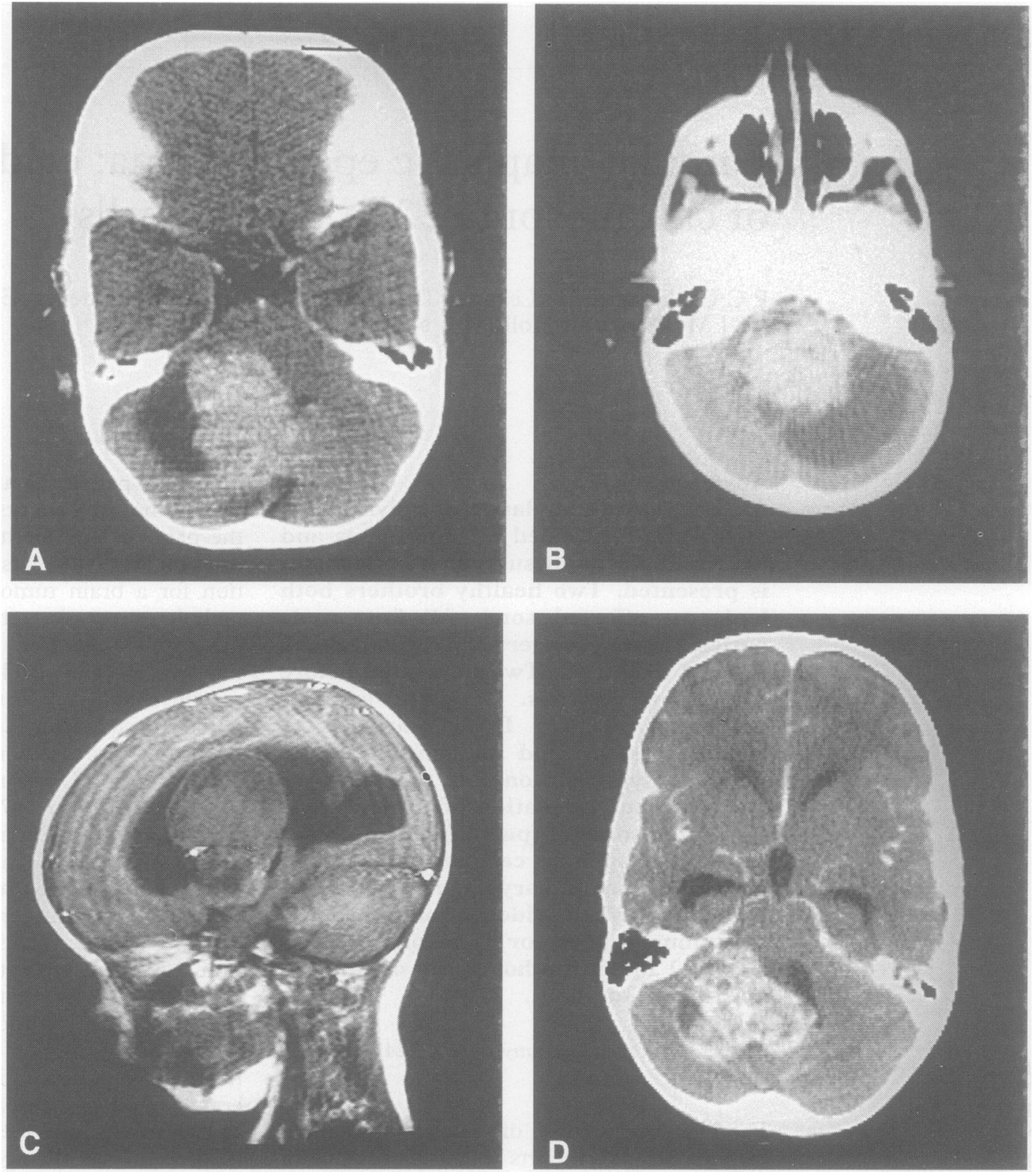

(fig 2B). Despite ventricular shunting he died several days later. Necropsy was not permitted and there is no histology of the tumour.

Patient C, born 1989, a first cousin of patients $A$ and $B$, was admitted at the age of 21 months with vomiting, lethargy, increasing skull circumference, and delayed development of motor skills. Computed tomography and MRI showed a large intraventricular supratentorial tumour with contrast enhancement (fig 2C). Angiography showed a large tumour blush with vascularisation mainly from choroid arteries. Craniotomy showed a highly vascular subependymal tumour. He died four months after the biopsy.

Patient D, born 1990, a brother of patient $C$, was followed up regularly because of the family history. At the age of 2 months cerebral CT was normal. At the age of 8 months he started vomiting and showed delayed motor skills. Computed tomography and MRI showed an infratentorial tumour with contrast enhancement (fig 2D). The tumour was surgically removed and used for genetic analysis. Postoperative treatment consisted of chemotherapy with vincristine, procarbazine, and methotrexate. At 2.5 years old a routine MRI showed a small recurrence of tumour at the operation site, which has been extirpated recently.

MICROSCOPIC EXAMINATION (PATIENTS A, C, AND D; FIG 3).

All tumours were well vascularised and highly cellular, with nuclear polymorphism, several mitoses, and irregular amounts of cytoplasm. Immunohistochemistry was positive for GFAP，EMA，NSE，S-100, LEU-7，LCA, vimentin, and $a_{1}$ antitrypsin, and negative for several other labels. In patient $C$ some cells were arranged in ribbons, and rosettes and microcystic spaces were found. In patient D typical tubuli and some necrosis were present. Electron microscopy of this tumour showed a uniform population of cells with well developed rough endoplasmatic reticulum and Golgi complex. Desmosomal structures and interdigitating villi were common. 

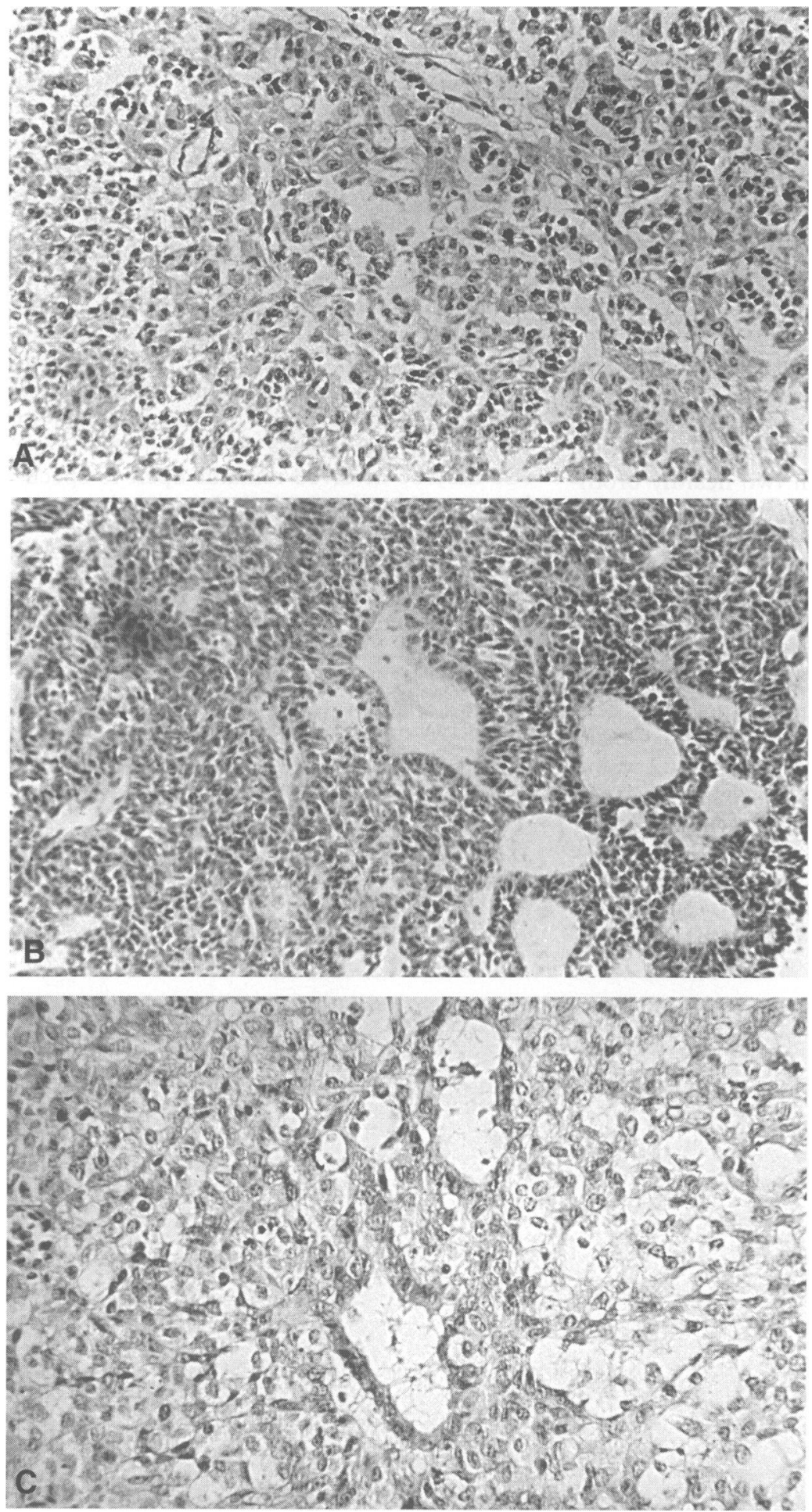

Figure 3 Histopathology (haematoxylin and eosin). (A) Patient A. (B) Patient B. (C) Patient $D$ (all magnifications originally $\times 25$ ).
D22S193, and cos 13. The probes are ordered from centromere to telomere. ${ }^{4}$

\section{INTERPHASE CYTOGENETICS}

Nuclei isolated from freshly removed tumour cells were investigated for aberrations of chromosomes 1, 6, 7, 10,11,17,18, 22, $\mathrm{X}$, and $\mathrm{Y}$ by fluorescent in situ hybridisation (FISH), according to Arnoldus et al..$^{5}$ In addition, we used probes specific for the short arm or the centromeric regions of chromosome 15 (D15Z1) and 22 (p22/1:2.1), and two cosmid probes ( $\cos 58$ and $\cos 13)$ that are located close to D22S193 on band 22q11. One hundred cells were counted for each probe. Cultured lymphocytes from blood of an unrelated subject were used as a control.

\section{Results}

Cytogenetic analysis of lymphocytes and fibroblasts in patients $C$ and $D$ respectively showed a normal male karyotype. The tumour of patient $\mathrm{D}$ was investigated in more detail. Interphase cytogenetic analysis performed on freshly removed tumour tissue with probes for chromosomes $1,6,7,10,11$, $15,17,18, \mathrm{X}$, and $\mathrm{Y}$ gave results in the normal range. This indicates a diploid tumour without gross aberrations of these chromosomes.

Additional techniques were applied to investigate chromosome 22 . Interphase cytogenetics on freshly removed tumour tissue showed only one fluorescent spot in about $76 \%$ of nuclei with three probes for chromosome 22 (table). Moreover, seven polymorphic probes for chromosome 22 were used to study possible loss of heterozygosity in tumour DNA. D22S193 was the only informative probe. This probe clearly showed loss of heterozygosity of allele 2 when DNA derived from freshly removed tumour tissue was compared with DNA from fibroblasts of the same patient. We also looked at mutations in the recently cloned NF2 gene by RNA SSCP analysis. There was no evidence for mutations in RNA from this tumour. The same method showed mutations in $36 \%$ of 53 meningiomas and schwannomas (RH Lekanne Deprez et al, unpublished data).

\section{Discussion}

The histology and immunohistochemistry, intraventricular location, age of onset, and identical neuroradiological patterns indicate that the tumours in all four patients are similar and of ependymal origin. Although they are not typical ependymomas, they lack the major criteria for ependymoblastomas. Considering age of onset, location, morphology and immunohistochemistry, we designated these tumours as anaplastic ependymomas.

Familial ependymal tumours are very rare, and to our knowledge only nine families have been reported. These reports include three families with two or more ependymal tumours, ${ }^{13}$ and five ependymal tumours asso- 
Table Interphase cytogenetic analysis of tumour of patient $D$

\begin{tabular}{lllll}
\hline & & \multicolumn{3}{c}{ Number of spots per nucleus } \\
\cline { 3 - 5 } Tissue & Probes & 0 & 1 & 2 \\
\hline Fresh tumour & $\mathrm{p} 22 / 1: 2 \cdot 1$ & 6 & 73 & 21 \\
& Cos 58 & 8 & 76 & 16 \\
Blood control $^{\star}$ & Cos 13 & 6 & 79 & 15 \\
& p22/1:2.1 & 7 & 10 & 83 \\
& Cos 58 & 8 & 13 & 79 \\
\hline
\end{tabular}

^ Cultured lymphocytes from blood of an unrelated subject.

ciated with glioma, plexus papilloma, and medulloblastoma. ${ }^{16}$ Although coincidence may account for part of these concurrences, the occurrence of four affected sons in a single generation, born from two healthy brothers, strongly suggests the involvement of a genetic predisposing mutation.

DNA and interphase cytogenetics of the tumour of patient D showed loss of (part) of chromosome 22 in a major percentage of tumour cells, indicating the involvement of a tumour suppressor gene. Although loss of chromosome 22 in tumour tissue might be a secondary effect of tumorigenesis, the consistent finding of loss of chromosome 22 in our and other reported cases, suggests a causal relation. Because ependymomas are sometimes found in neurofibromatosis type 2 , we searched for mutations in the NF2 gene in RNA from the tumour of patient $D$. No mutation was detected; thus we have no evi- dence that the NF2 gene is involved in this family. There are indications, however, for additional loci on chromosome 22 involved in the development of NF2 related tumours.

The likely transmission of the disorder by two healthy brothers, their exclusively male offspring, the occurrence of spontaneous abortions, and the history of a brain tumour in a paternal aunt, raise questions on the possible mode of inheritance. It cannot be explained by simple classic mendelian genetics, and variation in expression of an autosomal dominant gene is a proposed explanation, but lacks precision. Whether sex related effects of the transmitting parent (imprinting), or a gene with a variable insert size may be involved remains open to speculation, and awaits the isolation and characterisation of the gene involved.

1 Tijssen CC, Halprin MR, Endtz LJ. Familial brain tumours. The Hague: Martinus Nijhoff Publishers, 1982. Ranson DT, Ritland SR, Kimmel DW, et al. Cytogenetic and loss of heterozygosity studies in ependymomas, pylocytic astrocytomas and oligodendrogliomas. Genes pylocytic astrocytomas and oligoden

3 Savard ML, Gilchrist DM. Ependymomas in two sisters and a maternal male cousin with mosaicism with monoand a maternal male cousin with mosaicism with mono-
somy 22 in tumour. Pediatric Neuroscience 1989;15:80-4.

4 Biezen NA, van, Lekanne Deprez RH, Thijs A, et al. Isolation and characterisation of 25 unique DNA markers for human chromosome 22. Genomics 1993;15: 206-8.

5 Arnoldus EPJ, Noordermeer IA, Peters AC, et al. Interphase cytogenetics on brain tumors. Genes Chromosom Cancer 1990;3:101-7.

6 Sato T, Shimoda A, Taskahashi T, et al. Congenital anaplastic ependymoma: a case report of familial glioma. Childs Brain 1984;11:342-8. 